\title{
A position-sensitive Stroop effect: Further evidence for a left-to-right component in print-to-speech conversion
}

\author{
MAX COLTHEART, ANNA WOOLLAMS, SACHIKO KINOSHITA, and CONRAD PERRY \\ Macquarie University, Sydney, New South Wales, Australia
}

\begin{abstract}
In the classical Stroop effect, response times for naming the color in which a word is printed are affected by the presence of semantic, phonological, or orthographic relationships between the stimulus word and the response word. We show that color naming responses are faster when the printed word shares a phoneme with the color name to be produced than when it does not, in conditions where there is no semantic relationship between the printed word and the color name. This result is compatible with a variety of computational models of reading. However, we also found that these effects are much larger when it is the first phoneme that the stimulus and response share than when it is the last. Our data are incompatible with computational models of reading in which the computation of phonology from print is purely parallel. The dual route cascaded model computational model of reading, which has a lexical route that operates in parallel and a nonlexical route that operates serially letter by letter, successfully simulates this position-sensitive Stroop effect. The model also successfully simulates the "onset effect" in masked priming (Forster \& Davis, 1991) and the interaction between the regularity effect and the position in a word of a grapheme-phoneme irregularity (Rastle \& Coltheart, 1999b)-effects which, we argue, arise for the same reason as the position-sensitive Stroop effect we report.
\end{abstract}

In the standard version of the Stroop (1935) task, subjects are asked to name rapidly the colors in which visually presented stimuli are displayed. When these visual stimuli are themselves the written names of colors, the latency and accuracy of the color naming responses are affected by whether the written color name coincides with or differs from the color in which the stimulus is displayed and whose name must be produced as the response. Latencies are shorter and accuracy is higher when there is congruence than when there is conflict.

There are at least two processing loci where such Stroop effects could arise: semantic and phonological. Colorrelated words such as $s k y$ produce a Stroop effect relative to color-unrelated words such as put (Klein, 1964); that effect must be semantic in nature. Pronounceable nonwords such as dap produce a Stroop effect relative to random consonant strings such as fjq (Bakan \& Alperson, 1967); that effect is taken to be phonological in nature.

We are concerned here specifically with the phonological locus of Stroop effects. The results of Bakan and Alperson (1967) suggest that, even for nonwords, phonology is generated automatically from print, even when this is detrimental to performance, as in the Stroop situation. If so, the investigation of the phonological Stroop effect

We are grateful to Ken Forster for drawing our attention to the work of Dalrymple-Alford (1972) and for much helpful editorial comment, and to Kathy Rastle and Jo Ziegler for advice on the DRC and other models of reading. Correspondence should be addressed to $\mathrm{M}$. Coltheart, Department of Psychology, Macquarie University, Sydney NSW 2109, Australia (e-mail: max@currawong.bhs.mq.edu.au). might be another fruitful way of studying how print is converted to speech, a topic of perennial interest to students of the psychology of reading.

The dual-route framework (Baron \& Strawson, 1976; Coltheart, 1978; Forster \& Chambers, 1973) proposes a distinction between lexical and nonlexical procedures for converting print to speech. The lexical procedure depends on the looking up of preexisting lexical representations, and so such a procedure can work correctly only for words; the nonlexical procedure depends on application of grapheme phoneme correspondence rules, and so cannot work correctly for exception (irregular) words, but succeeds with regular words and pronounceable nonwords.

A specific computational implementation of the dualroute framework, the dual route cascaded (DRC) model, has recently been developed (Coltheart, Curtis, Atkins, \& Haller, 1993; Coltheart, Langdon, \& Haller, 1996; Coltheart \& Rastle, 1994; Rastle \& Coltheart, 1998, 1999a, 1999 b). Crucial for our present purposes is the claim in that model that the derivation of phonology from print occurs in parallel across letters on the lexical route, but is a left-to-right serial process across letters on the nonlexical route. Hence input to the phoneme level from the lexical route influences all phonemes simultaneously, while at the same time input to the phoneme level from the nonlexical route comes in from left to right; these two asynchronous sources of input are thus integrated in a single common set of phoneme units. One very strong prediction made from such a claim is this: For irregular words, the later the position in the word its irregular graphemephoneme correspondence is, the smaller will be the la- 
tency cost of its irregularity. Coltheart and Rastle (1994) and Rastle and Coltheart (1999b) have confirmed this prediction in human data, and have also shown that the same position-of-irregularity effect occurs in the naming latencies of the DRC model when it is reading the same words.

\section{The Onset Effect in Masked Form Priming}

Another phenomenon that we believe should be attributed to the left-to-right serial nature of nonlexical translation from print to speech is the onset effect (Forster \& Davis, 1991); hence we next briefly consider the nature of this effect and its simulation by the DRC model. When a lowercase prime that is briefly presented and then masked shares the same initial phoneme with an uppercase target that is presented subsequently for naming (belly-BREAK), naming latency for the target is reduced, relative to a no-similarity condition (merry-BREAK) or a rhyming condition (take-BREAK) (Forster \& Davis, 1991).

Forster and Davis (1991) offered a dual-route interpretation of this onset effect, attributing it to the influence of a nonlexical process that translates print to speech in a serial (left-to-right) order. The rate at which this serial process operates is such that, in the limited time for which the prime is presented, the process has generally translated only the first phoneme of the prime, and so priming due to this process occurs only when prime and target have a common first phoneme.

If this dual-route analysis is correct, it should be possible to simulate the onset effect with the DRC model; hence DRC simulations of the onset effect were run with the materials from Experiment 1 of Forster and Davis (1991). After a few items were discarded from these materials for various reasons - such as polysyllabicity (the DRC model is at present applicable to monosyllabic stimuli) - 18 prime-target pairs were used per condition. Priming was simulated by having the model process the prime for some number of processing cycles and then replacing the prime with the target, without resetting to zero the unit activations generated in response to the prime. The model's naming latency for each prime was measured.

When primes were run for 40 cycles (which is too short a period for DRC to name or identify them) and then the target was immediately presented for naming, the model always erred: Its naming response was always the name of the prime, not the name of the target. Even with a prime duration of 25 cycles, this happened on most occasions. The reason for this is that, because the primes are words, even 25 cycles is enough to build activations of the orthographic and phonological lexical entries for the prime up to a level where a new incoming stimulus cannot inhibit preexisting activations; instead, the preexisting (prime) activations inhibit activation of the target's lexical entries, and so the prime blocks the target and is incorrectly produced as the naming response.

Forster and Davis (1991) actually observed this phenomenon on a few occasions; that is, their subjects sometimes responded by naming the (invisible) prime rather than the target. But this occurrence was rare, and so the DRC simulations need to use a prime duration at which DRC's response is the target rather than the prime on almost all occasions. Once such a prime duration is found, the question is, will the onset effect be correctly simulated?

Since a prime duration of 25 cycles produces far too much blocking, one might be tempted to try, say, 10 cycles for prime duration. But, just as we can show that a prime duration of 25 is too long, so we can show that a prime duration of 10 cycles is too short, at least with the parameter set used to simulate various effects in reading aloud (Rastle \& Coltheart, 1998, 1999a, 1999b), because with this parameter set, input from the grapheme-phoneme correspondence (GPC) system to the phoneme level does not begin until Cycle 11; if the onset effect is due to the GPC system, then a prime duration of 10 cycles will not be long enough to produce the effect.

Given that the operation of the DRC model is controlled by a relatively large number of parameters, it might be argued that the DRC model is too powerful; that is, it "can explain everything." Here is a clear counterexample to such an argument. If we explore prime durations between 10 and 25 , as we increase prime duration, two things will happen. The longer the prime duration, the more activity the nonlexical route will be contributing to the first phoneme position, and so the more priming there will be when prime and target share this phoneme. But also, as we increase prime duration, the representations of the prime word at all levels of the lexical route of the DRC model will be strengthened, and so blocking will be more likely to occur. It is therefore not at all obvious that there will be a value of prime duration between 10 and 25 that is (1) long enough to cause a robust onset effect yet (2) not long enough to cause blocking. So it is not at all obvious that the model could successfully simulate the onset effect.

It turns out, however, that with a prime duration of 12 cycles, there is no blocking (all targets are named correctly), but there is a significant onset effect; DRC's target naming latencies are significantly faster in the primed (pole-PEAR) than in the control condition (take-PEAR). The model also showed a much smaller but significant priming effect in the rhyme prime condition (hair-PEAR); the 6-msec effect in this condition in the Forster and Davis (1991) experiment was not significant, and so further simulation and human-experimental work on this rhyme priming effect is needed. In the DRC model, the effect is not due to the rhyme relationship, but to the shared letter $r$ (since the model shows no priming with hare-PEAR).

It is easy to demonstrate that the onset effect in the DRC model arises at the phoneme level. At the end of the 12 th cycle (i.e., at prime offset), none of the target's phonemes have been activated except for the first phoneme of the target in the first-letter prime condition. For 17 of the 18 items, the first phoneme of the target has an activation of between .004 and .005 . This gives target naming its extra boost in the first-letter prime condition.

What about the one item where this does not happen? It is the pair know-KNEE. The reason that the prime know 
has not activated the phoneme / $\mathbf{n} /$ after 12 cycles is that at that cycle the left-to-right GPC process has identified only the first letter of the prime $(k)$ and has translated that to the phoneme $/ \mathrm{k} /$. This is the only prime-target pair in the first-letter prime condition that begins with a multiletter grapheme.

Hence the DRC model makes the following prediction: In a masked priming experiment with human subjects, the onset effect will be smaller (or even absent) for primetarget pairs that begin with multiletter graphemes (shopSHED, east-EACH, know-KNEE) than for pairs that begin with single-letter graphemes (stop-SLED, else-EBBS, king KEYS). If the onset effects in these two conditions do not differ in size, this would imply that the serial processing of the GPC system in dual-route models is phoneme by phoneme, not letter by letter, and so DRC's GPC procedure would have to be redesigned so that it operates phoneme by phoneme, rather than letter by letter. But in that case, the model would no longer be able to explain the "whammy effect" of Rastle and Coltheart (1998), which depends on the GPC procedure operating letter by letter. Thus strong predictions are being made here.

\section{A Serial Effect in the Stroop Task?}

We have seen that the claim that the nonlexical translation from print to speech operates serially left to right is supported by human and simulation data from studies of the regularity effect and the onset priming effect. This claim also makes a strong prediction about phonological Stroop interference. Suppose the stimuli in a Stroop experiment are words that are all semantically unrelated to colors, such as rat, pod, or kit. And suppose these three words are printed in red, so that for all three, the response the subject will utter is the word "red." Since the meanings of these words have nothing to do with colors, there will be no semantic Stroop effect - or, certainly, no difference between these words in the strength of such a semantic effect.

All three phonemes of the word kit clash with the corresponding phonemes of the correct response. But the words rat and pod each have one phoneme in common with the correct response. Might this one-phoneme overlap generate some benefit for the "red" response, relative to the no-phoneme overlap condition? If such a benefit is observed at all, then the prediction from the DRC model is that this benefit will be greater when the overlap is in the first phoneme position (rat) than when it is in the lastphoneme condition ( $p o d)$. Since the lexical route operates in parallel, it will make the relevant phonemes $/ \mathrm{r} /$ and $/ \mathrm{d} /$ available equally; but the simultaneously operating nonlexical route will make / $\mathbf{r}$ / available early in processing and /d/ available late in processing. That is why the response "red" should be faster with the red word rat than with the red word pod, if the DRC model is correct. Furthermore, the response "red" should be faster with the red word pod than with the red word kit (unless the left-to-right nonlexical route is operating so slowly that such items are color named before the nonlexical route has reached the third letter and begun to activate its phoneme).

\section{Previous Studies of \\ Positional Effects in the Stroop Task}

To the best of our knowledge, only three of the huge number of publications on the Stroop effect deal with anything that is relevant to these specific predictions about the effects of the position of phoneme overlap on the size of the phonological Stroop effect.

The first of these is the study by Dalrymple-Alford (1972). He reported that the color naming of words whose meanings were semantically unrelated to colors was faster when the pronunciation of the word shared some phonemes with the pronunciation of the correct color name (run or bed colored red compared with pun or cat in the same color). Dalrymple-Alford also manipulated whether the phonological similarity was "initial sound similar" or "final sound similar," but this was done unsystematically. For example, a word he classified as "initial sound similar" re the response "green" was grown, which also has a final letter/phoneme in common with the pronunciation "green"; and a word classified as "initial sound similar" re the response "red" was rid, which has a final phoneme in common with the pronunciation "red." Also, it was not just the first or the last "sound" (i.e., phoneme) that was similar, but the initial or final few phonemes. Furthermore, Dalrymple-Alford did not test statistically whether the size of the phoneme-overlap Stroop effect varied as a function of his initial-sound-similar versus final-soundsimilar variable, though the effect was bigger in the former condition ( 86 vs. $58 \mathrm{msec}$ ). Thus, although DalrympleAlford's data show that partial phonological overlap between word and color name can generate a Stroop effect, we cannot tell from the data whether the position of such an overlap makes a difference (or whether overlap of just one phoneme is sufficient to generate a Stroop effect).

Next is the study by Singer, Lappin, and Moore (1975), which more effectively addressed the position issue. Each trial of their experiment required the subject to name the ink color of all the items in a printed list of 100 items. There were seven conditions in this experiment. In Condition 1, all of the items were color names incongruent with their ink color (e.g., orange printed in brown). In Conditions $2-4$, the items were color names incongruent with ink color and with their middle or final letters deleted or altered (e.g., orxxge, or, and oranxx). In Conditions 5-7, the items were color names incongruent with ink color and with their initial or middle letters deleted or altered (e.g., xxange, an, and ge). Color naming was significantly slower in Condition 1 than in all of the other conditions. Conditions 2-4 did not differ from each other, but all three conditions were significantly slower than each of Conditions 5-7; these last three conditions did not differ significantly from each other. Thus when the printed 
stimulus shares some letters or phonemes with a color name that is not the one that should be named, there is more interference when the shared segment is at the beginning than when it is at the end.

The other relevant study, and the study most directly related to our work, is that of Regan (1978), which was not concerned with the position issue but did address the issue of whether overlap of a single phoneme (or letter) could generate a Stroop effect. In her Experiment 3, the stimulus was a single alphanumeric character whose color had to be named, the colors involved being red, green, and blue. Color naming latency did not differ between the condition where the item was a digit $(632 \mathrm{msec})$ and the condition where it was a letter that did not correspond to the initial letter or phoneme of any of the three color names $(636 \mathrm{msec})$. However, when the item contained the initial letter/phoneme of the color to be named, responses were significantly faster ( $574 \mathrm{msec}$ ) than in either of the control conditions, and when the item contained the initial letter/ phoneme of the color different from the one that was to be named, responses were significantly slower $(691 \mathrm{msec})$ than in either of the control conditions.

These studies show that when there is some orthographic or phonological correspondence between a part of the stimulus carrying the color and a part of the color name, Stroop effects can be generated. Only the Singer et al. (1975) work, however, provides evidence that the position of this overlap makes a difference, with a larger effect of overlap when the overlap is at the beginning than when it is at the end. The aim of our study was to seek to confirm this result using better controlled stimuli (in our study, overlap was always exactly one phoneme and this was always the first or the last phoneme) and using monosyllabic words so that a DRC simulation of the results could be carried out (the model currently reads only monosyllabic words).

\section{METHOD}

\section{Design}

The present experiment featured a 2 (overlap) $\times 2$ (position of overlap) $\times 3$ (color) fully repeated measures design. Each subject named the color of all the stimulus words, half of which possessed phonemic overlap with either the initial or final phoneme of the color names red, green, or blue.

\section{Subjects}

Undergraduate students $(N=24)$ from Macquarie University volunteered to participate in exchange for course credit. All subjects were native Australian-English speakers.

\footnotetext{
Materials

Ninety-two words possessing phonemic overlap with either the initial or the final phoneme of one of the three color names (red, green, blue) were selected as critical stimuli. The selection criteria for critical stimuli required them to range between 3 and 5 letters in length, to have the same number of phonemes as the color name with which they overlapped, to have an absence of phonemic overlap with any other phoneme of the three color names, and to have a lack of any obvious color association. Of the 92 critical stimuli selected, 20 began with $/ \mathrm{r} /$ (e.g., rack), 12 began with /g/ (e.g., games), 20 began with $/ \mathrm{b} /$ (e.g., back), 20 ended with $/ \mathrm{d} /($ e.g., cod), 12 ended
}

with $/ \mathbf{n} /$ (e.g., scan), and 8 ended with $/ \mathrm{u} /($ e.g., cue $) .{ }^{1}$ Each of these words was presented only in the color with which it overlapped phonemically; that is, only congruent Stroop trials were included in the present experiment. A further 92 words not possessing any phonemic overlap with any of the three color names were selected as control stimuli. These control stimuli were matched to the critical stimuli on number of letters and number of phonemes. Each control stimulus was presented in the same color as its matched critical stimulus. A complete list of stimuli appears in the Appendix.

These 184 items were submitted to a version of the DRC model equipped with a semantic system containing semantic entries for the three relevant words red, green, and blue. These items were also used in a color-naming experiment with human subjects.

There were stringent phonological and orthographic constraints on stimulus selection here. Hence it was impossible to match items exactly on two conceivably relevant variables: $\log$ word frequency (Baayen, Piepenbrock, \& van Rijn, 1993) and N (neighborhood size) of the stimulus words. We therefore dealt with potential confoundings on these two variables by statistical means - by using analyses of covariance with the two variables as covariates. These analyses of covariance yielded exactly the same patterns of significance and nonsignificance as conventional analyses of variance; hence we report the latter analyses.

\section{Procedure}

Stimuli were displayed on an NEC Multisync 4FG monitor, controlled by a Deltacom 386 IBM-compatible computer. Stimuli were presented in a different random order to each subject. Subjects were instructed to name the color of the letter strings aloud as quickly as possible. A series of six control stimuli (two of each color) were then presented as practice trials.

Instructions and stimuli were presented and reaction time data recorded by means of the DMASTR display system (Forster \& Forster, 1990). Naming latency was recorded using a voice key individually adjusted for each subject and held a constant distance away from the mouth throughout the experiment by a voice key headset. Error rates, error types, and measurement errors resulting from inaccurate voice key activation were recorded manually by the experimenter.

Presentation of each stimulus item was preceded by a white fixation asterisk appearing in the center of the screen for $497 \mathrm{msec}$. The stimulus item then appeared in lowercase in the center of the screen in the appropriate color, and remained on the screen for a maximum of $2,000 \mathrm{msec}$. The stimulus item was replaced by a blank screen as soon as the subject initiated articulation of the color name. Once the subject had completed articulation of the color name, the fixation asterisk for the next stimulus item appeared after a delay of $994 \mathrm{msec}$.

\section{RESULTS}

\section{Simulation Results}

Although we provided above a brief discussion of what the DRC model as extended to the Stroop color naming task predicts about the results of our experiment, it is important to recognize that to determine by reasoning alone exactly what any serious computational model predicts about anything is a complex task. It is very easy to overlook aspects of the way complex computational models work when reasoning in this way, and so easy to err in reaching conclusions about exactly what such models predict. The only safe course of action here is actual simulation. Therefore we had to extend the DRC model of reading in such a way as to allow simulation of color naming. We did this by adding a minimal semantic system containing semantic units just for the words red, green, 


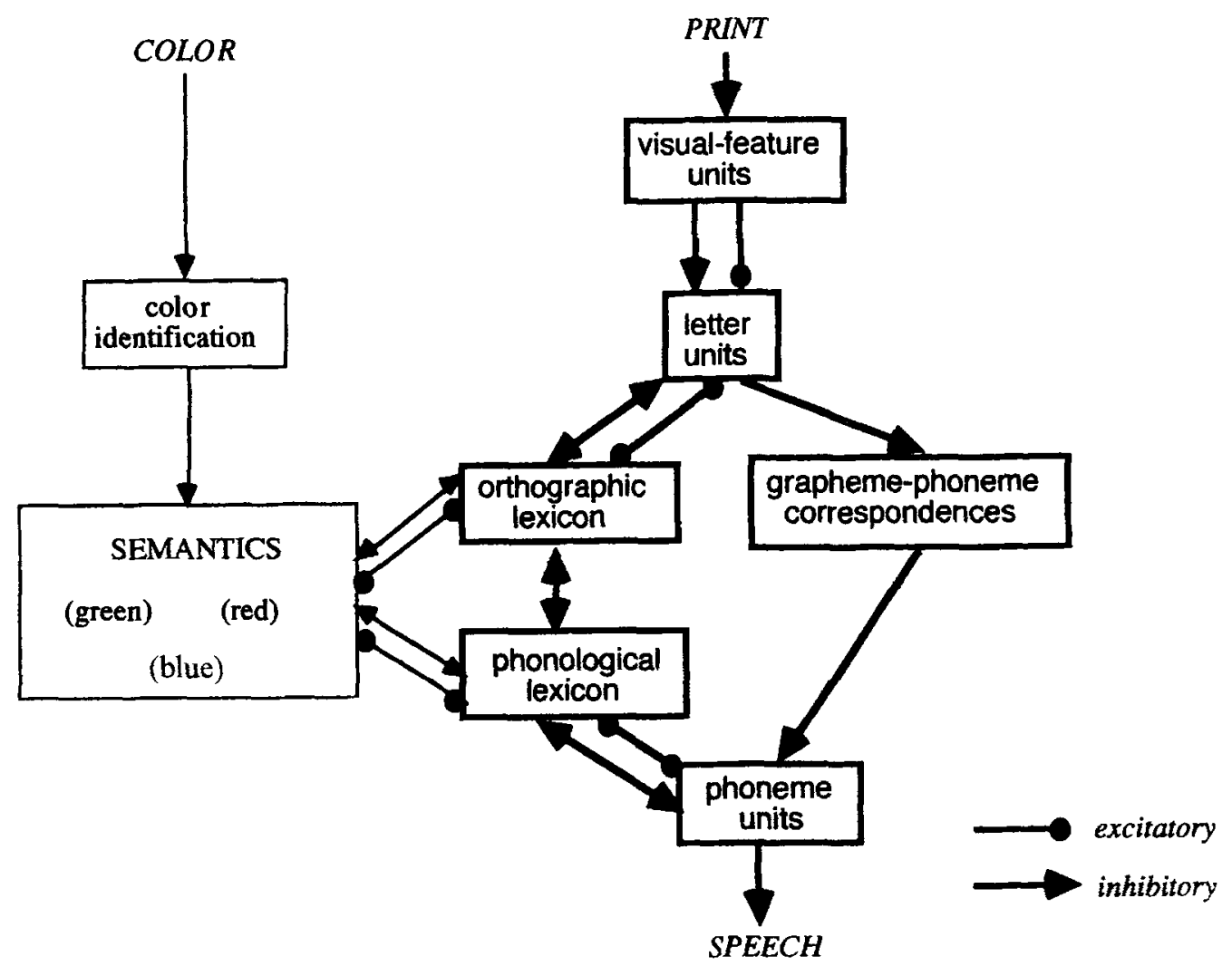

\begin{abstract}
Figure 1. Extension of the DRC model for analysis of Stroop color naming with printed stimuli. Implemented components of the model are shown by heavy lines. The semantic system is currently unimplemented except for some color concepts as described in this paper.
\end{abstract}

and blue. Each of these semantic units was connected to its phonological representation in DRC's phonological output lexicon by excitatory connections, and there were excitatory connections in the reverse direction. The strengths for each set of excitatory connections were set to 1.0. There were two other parameters associated with this semantic system. The first was a semantic activation parameter that controlled the amount of activation in the semantic units: The activation of these units grew as a logistic function of processing cycles, and the actual activation of a unit on any cycle was the value of this function multiplied by the activation parameter. We used a value of .58 for that parameter. Finally, there was a delay parameter: Activation did not begin to rise in the semantic system until the number of cycles corresponding to the delay parameter had elapsed. This is a crude way of simulating the time required by presemantic color identification processes. We set this delay to 82 cycles. The DRC model with this minimal semantic system is shown in Figure 1.

On any cycle of this extended model, the activation levels of the phonemic units were updated in two ways. The phonemes activated from the letter string via the lexical and the nonlexical routes were updated in exactly the same way as when DRC's task is reading aloud. But in addition, the phonemes corresponding to the desired color name were updated, too, via the route from semantic system to phonological output lexicon to phoneme units. Thus there was phoneme activation corresponding to the word being presented and also to the color being presented.

Now, of course, people presented with a word printed in a color are capable of either naming the color or reading the word, depending on instructions. We have proposed elsewhere (Rastle \& Coltheart, 1999b) that strategic effects in reading-aloud tasks are most naturally simulated in terms of strategic adjustment of the strength of particular sets of connections in the model, and we adopt the same approach to simulating task instruction effects. If the task is color naming, the reading-aloud response should be weakened by turning down or off a parameter relevant to reading aloud; if the task is reading aloud, the color naming response should be weakened by turning down or off a parameter relevant to color naming. With the extended DRC model, if all of the parameters of the model except the new semantic parameters are given exactly the same values as used in various previous studies of simulated reading aloud (Rastle \& Coltheart, 1998, 1999a, 1999b), the model conflates the two tasks; its response is often a mixture of phonemes from the color name and the word (such as responding to the word sad written in red as "/sEd/"). We simulated the instruction, 
Table 1

Mean Correct DRC Color Naming Latencies as a Function of Phonemic Overlap and Position of Phonemic Overlap

\begin{tabular}{ccc}
\hline & \multicolumn{2}{c}{ Phonemic Overlap } \\
\cline { 2 - 3 } Item & Initial & Final \\
\hline Critical & 127.55 & 131.48 \\
Control & 131.76 & 132.54 \\
\hline
\end{tabular}

"Name the color, don't read the word" by setting to zero the connections from the letter level to the orthographic input lexicon. ${ }^{2}$ When this was done, the extended model produced the correct color name as its response for 180 of the 184 items ( $97.83 \%$ correct); all four wrong color naming responses consisted of initial phonemes from the stimulus word followed by subsequent phonemes from the color name (e.g., had in red yielded the response "/hEd/").

The four wrongly named items and their matched control words were discarded before analysis of the correct DRC color naming latencies using a $2 \times 2 \times 3$ analysis of variance (ANOVA), with phonemic overlap (critical vs. control) treated as a within-items factor and both position of phonemic overlap (first vs. final) and color (red, green, blue) treated as between-items factors. Mean correct DRC color naming latencies for critical stimuli possessing initial or final phoneme overlap (collapsed across color since the color variable did not interact with any other variable) are presented with those for matched control stimuli in Table 1.

As can be seen in Table 1, a substantial priming effect resulted from phonemic overlap with the color names. This advantage for stimuli possessing phonemic overlap with the color names was highly significant $[F(1,82)=95.45$, $p<.0005]$.

Moreover, the magnitude of the phonemic overlap priming effect was influenced by the position of phonemic overlap, as indicated by a significant interaction between these two factors $[F(1,82)=33.97, p<.0005]$. Inspection of Table 1 shows the form of this interaction: The phonemic overlap priming effect was more pronounced for items with initial position phonemic overlap than for items with final position overlap. However, the phonemic overlap effect was significant at both positions; at initial position, the effect was 4.21 cycles $[F(1,47)=99.59, p<$ $.0005]$, and at final position, it was 1.06 cycles $[F(1,35)=$ $19.29, p<.0005]$.

Analyses also revealed a reliable main effect of color $[F(2,82)=5.58, p=.005]$. This difference in color naming latencies did not interact with either the phonemic overlap priming effect $[F(2,82)=0.37, p=.694]$ or the observed asymmetry of the phonemic overlap priming effect according to the position of phonemic overlap $[F(2,82)=$ $0.94, p=.394] .^{3}$

\section{Human Data}

Any trial on which a subject or voice key error occurred was excluded from the calculation of mean naming laten- cies for analysis, as was its matched item. Errors occurred on only $2.85 \%$ of trials. These errors resulted from inappropriate voice key activation or perseverative production of an incorrect color name. In order to reduce the effects of outliers, naming latencies more than $2 S D$ from each subject's mean were replaced with the cutoff value. Greenhouse-Geisser corrected critical values are given where appropriate.

For the subjects analysis, the mean correct color naming latencies were analyzed by a $2 \times 2 \times 3$ ANOVA with phonemic overlap (critical, control), position of phonemic overlap (initial, final), and color (red, green, blue) all treated as within-subjects factors. For the items analysis, phonemic overlap was treated as a within-items factor, while both position of phonemic overlap and color were treated as between-items factors. Mean correct color naming latencies for critical stimuli possessing initial or final phoneme overlap are presented with those for matched control stimuli in Table 2.

As can be seen in Table 2, a substantial priming effect resulted from phonemic overlap with the color names. This advantage for stimuli possessing phonemic overlap with the color names was highly significant across both subjects and items $\left[F_{\mathrm{s}}(1,23)=36.93, p<.0005 ; F_{\mathrm{i}}(1,86)=\right.$ $46.01, p<.0005]$.

Moreover, the magnitude of the phonemic overlap priming effect was influenced by the position of phonemic overlap, as indicated by a significant interaction between these two factors $\left[F_{\mathrm{s}}(1,23)=4.89, p=.037 ; F_{\mathrm{i}}(1,86)=\right.$ $7.94, p=.006]$. Inspection of Table 2 shows the form of this interaction: The phonemic overlap priming effect was more pronounced for items with initial position phonemic overlap than for items with final position overlap. However, the phonemic overlap effect was significant at both positions. At initial position, the effect was $30 \mathrm{msec}$ $\left[F_{\mathrm{s}}(1,23)=30.75, p<.0005 ; F_{\mathrm{i}}(1,49)=44.99, p<.0005\right]$, and at final position it was $12 \mathrm{msec}\left[F_{\mathrm{s}}(1,23)=6.23, p=\right.$ $.020 ; F_{\mathrm{i}}(1,37)=9.74, p=.003$.

Analyses also revealed a reliable main effect of color $\left[F_{\mathrm{s}}(2,46)=40.26, p<.0005 ; F_{\mathrm{i}}(2,86)=64.16, p<.0005\right]$. This difference in color naming latencies did not interact with either the phonemic overlap priming effect $\left[F_{\mathrm{s}}(2,46)=\right.$ $\left.1.19, p<.308 ; F_{\mathrm{i}}(2,86)=1.18, p=.312\right]$ or the observed asymmetry of the phonemic overlap priming effect according to the position of phonemic overlap $\left[F_{\mathrm{s}}(2,46)=\right.$ $\left.0.29, p=.750 ; F_{i}(2,86)=0.23, p=.796\right]$.

These analyses indicate that the human and DRC simulation data agree very closely. Both for people and for the model, color naming of printed words is faster when word and color share a phoneme than when they do not,

Table 2

Mean Correct Human Color Naming Latencies as a Function of Phonemic Overlap and Position of Phonemic Overlap

\begin{tabular}{ccc} 
& \multicolumn{2}{c}{ Phonemic Overlap } \\
\cline { 2 - 3 } Item & Initial & Final \\
\hline Critical & 557 & 571 \\
Control & 587 & 583 \\
\hline
\end{tabular}


and the size of this effect is larger when the shared phoneme is the first phoneme of the color name than when it is the last; the effect however, is still significantly greater than zero in the last-phoneme condition.

\section{CONCLUSIONS}

We take our results as evidence in support of the DRC model of reading and as evidence against models of reading that claim that the processes by which print is converted to speech are exclusively parallel (e.g., Plaut, McClelland, Seidenberg, \& Patterson, 1996; Zorzi, Houghton, \& Butterworth, 1998). Although such models are certainly compatible with the finding that color naming of printed stimuli benefits from a single letter/phoneme overlap between printed stimulus and color name, they are incompatible with the finding that this benefit is greater for initial than for final overlap. ${ }^{4}$ We believe that such models are also incompatible with the onset effect observed in masked priming studies, and with the interaction between regularity and position of irregularity in studies of naming latency. In contrast, all three effects can be readily accounted for by the DRC model.

\section{REFERENCES}

Batyen, R. H., Piepentrock, R., \& van Rijn, H. (1993). The CELEX lexical database [CD-ROM]. Philadelphia: University of Pennsylvania, Linguistic Data Consortium.

Bakan, P., \& Alperson, B. (1967). Pronounceability, attensity and interference in the color-word test. American Journal of Psychology, 80, 416-420.

Baron, J., \& Strawson, C. (1976). Use of orthographic and wordspecific knowledge in reading words aloud. Journal of Experimental Psychology: Human Perception \& Performance, 2, 386-393.

Coltheart, M. (1978). Lexical access in simple reading tasks. In G. Underwood (Ed.), Strategies of information processing (pp. 151216). London: Academic Press.

Coltheart, M., Curtis, B., Atkins, P., \& Haller, M. (1993). Models of reading aloud: Dual-route and parallel-distributed-processing approaches. Psychological Review, 100, 589-608.

Coltheart, M., Langdon, R., \& Haller, M. (1996). Computational cognitive neuropsychology. In B. Dodd, L. Worrall, \& R. Campbell (Eds.), Models of language: Hlluminations from impairment (pp. 936). London: Whurr.

Coltheart, M., \& Rastle, K. (1994). A left-to-right serial process in reading aloud. Journal of Experimental Psychology: Human Perception \& Performance, 20, 1197-1211.

DALRYMPLE-ALFORD, E. C. (1972). Sound similarity and color-word interference in the Stroop task. Psychonomic Science, 28, 209-210.

Forster, K. I., \& Chambers, S. (1973). Lexical access and naming time. Journal of Verbal Learning \& Verbal Behavior, 12, 627-635.

Forster, K. I., \& DAVIS, C. (1991). The density constraint on formpriming in the naming task: Interference effects from a masked prime. Journal of Memory \& Language, 30, 1-25.

FORSTER, K. I., \& FORSTER, J. C. (1990). User's guide to the DMASTR display system. Tucson: University of Arizona.

Kawamoto, A., Kello, C., Jones, R., \& Bame, K. (1998). Initial phoneme versus whole word criterion to initiate pronunciation: Evidence based on response latency and initial phoneme duration. Journal of Experimental Psychology: Learning, Memory, \& Cognition, 24, 862-885.

KLEIN, G. S. (1964). Semantic power measured through the interference of words with color-naming. American Journal of Psychology, 77, 576-588.

Plaut, D. C., McClelland, J. L., Seidenberg, M. S., \& Patterson, K.
(1996). Understanding normal and impaired word reading: Computational principles in quasi-regular domains. Psychological Review, 103, 56-115.

Rastle, K., \& Coltheart, M. (1998). Whammy and double whammy: Length effects in nonword naming. Psychonomic Bulletin \& Reviews, 5, 277-282

Rastle, K., \& Coltheart, M. (1999a). Lexical and nonlexical phonological priming. Journal of Experimental Psychology: Human Perception \& Performance, 25, 461-481.

Rastle, K., \& Coltheart, M. (1999b). Serial and strategic effects in reading aloud. Journal of Experimental Psychology: Human Perception \& Performance, 25, 482-503.

Rastle, K., Harrington, J, M., Coltheart, M., \& Palethorpe, S. (in press). Reading aloud begins when the computation of phonology finishes. Journal of Experimental Psychology: Human Perception \& Performance.

REGAN, J. (1978). Involuntary automatic processing in color-naming tasks. Perception \& Psychophysics, 24, 130-136.

Singer, M. H., LapPin, J. S., \& Moore, L. P. (1975). The interference of various word parts on color naming in the Stroop test. Perception \& Psychophysics, 18, 191-193.

STROOP, J. R. (1935). Studies of interference in serial verbal reactions. Journal of Experimental Psychology, 18, 643-662.

Zorzi, M., Houghton, G., \& ButterworTh, B. (1998). Two routes or one in reading aloud: A connectionist dual-route model. Journal of Experimental Psychology: Human Perception \& Performance, 24, $1131-1161$.

\section{NOTES}

1. For the blue-final-phoneme condition, it was necessary to include three four-phoneme words in order to obtain sufficient trials for statistical analysis.

2 . This is only one of a number of possible ways of simulating this instruction. Another is to set to zero the connections from orthographic lexicon to phonological lexicon. That would have the advantage that there would still be activation from orthographic lexicon to semantics, and so there would be scope for interference due to the meaning of the word, which could not happen if there were no activation of the orthographic lexicon, but which of course is observed. We could approach this by simply reducing, rather than setting to zero, the strengths of the connections from letter level to orthographic level. Yet another possibility is to allow entries in the orthographic lexicon to be activated from print, but to have their activation decay away after reaching some criterion; if the color naming response is generated after enough lexicalorthographic decay, that may allow the correct response to be made. We are currently exploring all these and other possibilities in further simulations of the Stroop effect with the extended DRC model.

3. The main effect of position of overlap and its interaction with color are not reported, since the meaning of the position of overlap variable is unclear when collapsed across overlap-only half of the items vary on the critical dimension.

4. Kawamoto, Kello, Jones, and Bame (1998) have suggested that data such as ours and those of Rastle and Coltheart (1999b) might be reconciled with purely parallel models of reading aloud by assuming that in speeded reading-aloud tasks, subjects might utter the initial phoneme of the stimulus, and thus trigger the voice key, before knowing what the subsequent phonemes are (except in the case where the initial phoneme is a plosive). Kawamoto et al. acknowledged that, if this were the case, the kinds of coarticulation effects normally seen in speech production could not occur, since these are effects of subsequent phonemes on prior ones. They therefore predicted that in speeded reading-aloud tasks, initial phonemes would not show coarticulatory effects generated by subsequent phonemes. Rastle, Harrington, Coltheart, and Palethorpe (in press) disconfirmed this prediction: In a standard naming latency experiment that yielded a standard result (a regularity effect on naming latency), they made detailed measurements of the articulatory gestures generating the initial phoneme of the reading-aloud response and found clear evidence of coarticulatory effects in the production of this initial phoneme. 
APPENDIX

Words Used in the Experiment

\begin{tabular}{|c|c|c|c|c|c|}
\hline \multicolumn{2}{|c|}{ Red } & \multicolumn{2}{|c|}{ Green } & \multicolumn{2}{|c|}{ Blue } \\
\hline Critical & Control & Critical & Control & Critical & Control \\
\hline rack & chap & games & moist & back & mock \\
\hline ram & hip & gapes & chimp & bait & tack \\
\hline rap & fit & gaps & swim & bash & tiff \\
\hline rash & stay & gasp & post & bat & pit \\
\hline rat & tip & gates & toast & batch & faith \\
\hline rays & wash & ghost & whisk & bath & path \\
\hline rich & kiss & gift & skip & bit & zip \\
\hline riff & cows & goats & stick & boat & sash \\
\hline rim & pot & gums & mats & book & foot \\
\hline rip & hat & gunk & sack & boss & mitt \\
\hline roach & thick & gust & sift & botch & shack \\
\hline roam & sham & guts & $\cos t$ & both & pass \\
\hline roars & shock & scan & twit & boys & toss \\
\hline rock & miss & scorn & shift & buck & chip \\
\hline rook & soot & skin & camp & buff & chat \\
\hline rot & tap & span & soft & bus & wit \\
\hline ruck & shop & spawn & coats & bush & whim \\
\hline rum & spa & spin & pips & but & jot \\
\hline rush & whip & spurn & hoist & buys & sway \\
\hline rut & vat & stain & kicks & buzz & fish \\
\hline $\operatorname{cod}$ & sat & stun & spit & cue & sap \\
\hline cud & fat & swan & wasp & few & cat \\
\hline $\mathrm{fad}$ & $\cot$ & sworn & skim & hue & top \\
\hline had & pop & twin & foams & pew & hit \\
\hline hid & $\operatorname{sip}$ & & & skew & fist \\
\hline hood & hook & & & spew & vast \\
\hline kid & mop & & & stew & stop \\
\hline $\operatorname{mad}$ & cap & & & view & mass \\
\hline maid & sick & & & & \\
\hline mud & hop & & & & \\
\hline pad & spy & & & & \\
\hline paid & soap & & & & \\
\hline pod & $\operatorname{map}$ & & & & \\
\hline sad & kit & & & & \\
\hline shod & cash & & & & \\
\hline thud & wish & & & & \\
\hline toad & vows & & & & \\
\hline void & moth & & & & \\
\hline wad & ham & & & & \\
\hline wood & cook & & & & \\
\hline
\end{tabular}

(Manuscript received April 29, 1998;

revision accepted for publication November 12, 1998.) 